\title{
RMIT Together: \\ An Integrated Mental Health Promotion Campaign \\ Designed to build Wellbeing and Connection \\ During the COVID-19 Pandemic
}

\author{
Bronwyn Gresham \\ Senior Advisor, Mental Wellbeing Initiatives \\ Vanna Garrick \\ Program Lead, Mental Wellbeing Initiatives \\ Lisa Chee \\ Student Training Coordinator \\ Cassandra Scott \\ Student Communications Advisor \\ RMIT
}

\begin{abstract}
With the unfolding COVID-19 pandemic, physical distancing requirements and new psychosocial risks, the RMIT Wellbeing team and leaders in the community focussed on responding to the changing needs of students and staff. Communication of reliable, timely and evidence-based information through the lens of mental health and wellbeing was a top priority. The 'RMIT Together' campaign was designed to bring the whole community together, creating new online groups to foster peer connection and coping skills development. This case study demonstrates the various functions and wellbeing benefits that mental health and wellbeing promotion, prevention and education can play during periods of unforeseen life-changing global stress. It also demonstrates the sustainability of such interventions to build community resilience and mental wellbeing.
\end{abstract}

\section{Keywords}

Mental health promotion, student mental wellbeing, COVID-19 support, psychosocial risks, social media

\section{Introduction}

RMIT is a multisector university of technology, design and enterprise, with a staff and student global community of approximately 100,000 members.

Guided by a whole of university Mental Wellbeing Strategic Action Plan (2020-2025) RMIT Wellbeing collaborated with work areas across the university to respond to the pandemic and deliver a whole of university mental health promotion campaign, RMIT Together, designed for both students and staff. The campaign was participatory, and invited students and staff to connect via new online groups. The goal was to build individual and collective protective factors for mental wellbeing within the learning and working RMIT community. By necessity, in response to the pandemic, the RMIT learning and support community had translated to almost total online engagement and interaction.

This article outlines the design of the RMIT Together campaign and provides a deeper look at the student-focussed elements, sustained online platforms and details the impact and outcome derived through this whole of community intervention.

\section{Mental wellbeing during COVID-19}

The mental health and wellbeing of the Australian population and university students alike has been of growing concern for many years. In the general Australian population, one in five adults experience a mental health condition. McGorry (2019) states that seventy-five percent of mental health challenges start during adolescence and the early years of adulthood (15-24 age range). This life stage is when many begin vocational and tertiary education. 
Some groups of students are at higher risk of mental health challenges. At risk groups include young people (aged up to 25 years), international students, students from low socio-economic backgrounds, $\mathrm{PhD}$ students and students with a disability (Orygen, 2020). Further, some common university experiences increase risk of significant stress such as financial stress, a sudden increase in autonomy and responsibility, and pressure to excel academically (Orygen, 2020). It was anticipated that these student groups and stress factors would be especially impacted by the consequences of the pandemic with the requirement for physical distancing, remote study and work, loss of employment and restrictions on access to family, supports and prior routines and structure.

The National Mental Health and Wellbeing Pandemic Response Plan (Mental Health Commission, 2020) presented early findings of the mental health impacts to the Australian population. It outlined a study revealing that the general community was reporting feelings of despair, fear, anger, boredom, loneliness, anxiety and increased levels of stress between March and April 2020.

\section{Bringing RMIT together in challenging times}

RMIT's immediate response was to embed clear wellbeing messaging through communications during COVID-19. This messaging highlighted the importance of mental health and support seeking.

The RMIT Wellbeing team gained leadership endorsement to rapidly create a whole of university mental health promotion campaign. Named RMIT Together, the campaign was designed to align with the Australian Government \#Inthistogether COVID-19 mental health promotion campaign (National Mental Health Commission, 2020). A short video was produced featuring the Vice Chancellor and RMIT Wellbeing Senior Advisor to demonstrate strong leadership coupled with expertise as the foundation of the campaign.

RMIT Together focussed on four key protective psychosocial factors for students and staff:

- Culture: build a community of care and compassion.

- Connection: provide meaningful opportunities for engagement to neutralise isolation and activate peer-to-peer support.

- Mental health literacy: offer reliable and timely evidence-based communications on mental health and wellbeing in the context of remote work and study during COVID-19.

- Psychosocial skills: offer evidence-based strategies and share experiences that promote mental wellbeing, particularly during times of challenge.

An evidence-based mental health promotion model for the general population, called the Five ways to wellbeing, was chosen as the foundation for literacy and skills. The rationale was to create consistent and evidence-based messaging and deepen targeted psychosocial skills. The Five ways to wellbeing features five evidence-based action areas that improve and protect mental health (Aked et al, 2008; Aked \& Thompson, 2011). It's analogous to the five food groups for healthy eating, but for mental health and wellbeing. The five action areas are: connect (relationships), take notice (being mindful), be active (physical health and movement), give (contribute and participate), keep learning (embrace new experiences and develop skills). The model has been implemented by governments e.g. New Zealand, and health services e.g. The Royal Melbourne Hospital, both of which were sources of inspiration.

RMIT Together launched in May 2020 and used all university communications channels so as to reach all students and staff over a two-week period. A campaign webpage and social media content was developed. Students received two electronic direct mails (EDMs) with clear calls to action and information on support services.

The first EDM focussed on culture and connection. This included a video from the Vice Chancellor and prompts to visit the webpage and join the RMIT Together online social group (Facebook group for students). 
The second EDM focussed on mental health literacy and psychosocial skills. This included an introduction to the Five ways to wellbeing model, prompts to join the RMIT Together group and an invitation to complete the RMIT online wellbeing module.

RMIT staff received tailored information via the emailed staff news bulletin and the staff intranet home page. Staff were invited to join an RMIT Together online social group (Yammer), and to explore the Five ways to wellbeing resources, wellbeing modules and supports.

In the two-week campaign period 6,070 students and 895 staff clicked through to the campaign pages. Students joined the RMIT Together Facebook group $(\mathrm{n}=2,329)$ and 300 staff joined the Yammer group. After these collective responses it was considered that RMIT Together had been successfully launched.

Attention then turned to engagement, sustainability and evaluation.

\section{A closer look at RMIT Together student-focussed elements}

\section{Phase 1. Design and collaboration}

RMIT Wellbeing developed the RMIT Together Facebook group in collaboration with university Communications Advisers and guided by student input. It is a moderated formal RMIT social media channel. As such, members must show that they are an RMIT student, staff or alumni and agree to the group guidelines.

A moderation team was established comprising of Wellbeing and Communications staff representatives and student interns. The initial focus was on the mechanisms required to ensure the safety and effective functioning of the group. The team developed a risk management plan, moderation guidelines, an evaluation framework logic model (Ebener, et al. 2017) and content themes were planned. Initially the moderation team met daily, then transitioned to twice-weekly meetings once the Facebook group was established. Meetings included strategic content planning and discussing moderation matters such as post approvals, new member requests, and content review.

The moderation team also communicated with stakeholders such as the Student Union, Student Services, representatives from marketing and with various academic schools and residential colleges.

\section{Phase 2. Growth and sustainability}

\section{Building membership}

Following the campaign period, the RMIT Together group was regularly promoted to build membership. Invitations to join were embedded across standard university channels and aligned with the rhythm of the university academic cycle and events calendar e.g. orientation, study week, university mental health day, graduation.

\section{Fostering engagement}

A key intention of establishing the Facebook group was to give students a felt experience of engagement. This included opportunities to participate and feel connected. Engagement with social media begins with viewing posts, "liking" viewed posts, commenting on or sharing posts, and initiating posts. Strategies used to move members through these stages included designing posts that require member engagement, such as asking questions, creating polls, and running competitions. Moderators encouraged students to post their own work and creative ideas relating to study and wellbeing during COVID-19. Content for competitions was designed to build psychosocial skills based in the Five ways to wellbeing model. Given the significant financial hardship experienced by students, prizes connected to the competitions met student needs, such as food support vouchers. 


\section{Strengthening wellbeing}

The content strategy for strengthening wellbeing skills and experiences was based on the Five ways to wellbeing model. This model became a foundation for content ideas and focussed periods of time in the university life cycle, such as during assessment and exam periods, and national days such as University mental health day.

Additional approaches to strengthen mental health knowledge, skill acquisition and practise included weekly livestreams and the promotion of RMIT Wellbeing's online course. The weekly livestreams occurred during Semester One through the facebook group and were presented by RMIT Wellbeing's clinical psychologist. The focus was on applying the Five ways to wellbeing model action areas to topics raised in the group. Livestream topics included study stress, coping with overwhelm, isolation and loneliness, self-care, sleep routines, mindfulness and compassion.

The RMIT Wellbeing online course teaches students about mental wellbeing during study, providing information on how to monitor for signs of stress and distress, some practical tips for supporting others, and lots of emphasis on self-care using strategies linked to the Five ways to wellbeing.

\section{Phase 3. Evaluation}

The RMIT Together Facebook group was evaluated through two mechanisms. The first was a monthly communications dashboard reporting on available metrics.

This included the number of group members, membership percentage increase, engagement rate percentage, number of posts, number of reactions (likes, comments, shares), top posts and top engagers.

The second mechanism was a wellbeing survey, administered to RMIT Together Facebook group members at the end of Semester One and Two. The wellbeing survey asked about the student user experience and attempted to draw out wellbeing related outcomes which resulted primarily from their involvement in the Facebook group and its related content.

Analysis of communications data and wellbeing survey responses in Semester One allowed the moderation team to test new approaches in Semester Two. This noticeably boosted group engagement rates in the latter part of 2020.

\section{Membership and engagement}

Within the first month the RMIT Together Facebook group had grown to 2281 members. Over the eight-month period to December 2020, the average monthly membership increase was $6 \%$, reaching a total of $>3000$ RMIT student members.

Engagement rates within the RMIT Together Facebook group averaged 20\%, meaning out of all of our group members, on average each month $20 \%$ engaged with the content (Table 1). This is significantly above the sector norm $(0.2 \%)$ for a tertiary sector social platform. Engagement rate is a metric used to assess the average number of interactions the social media content receives per follower. Here, it was calculated as the total number of interactions the content received divided by total number of followers that month, multiplied by $100 \%$. In simpler terms, it's a measure of how well the content performs independent of follower count.

Part of the RMIT Together strategy was to increase active engagement, so the number of posts, reactions and comments were also tracked (Table 1). Many posts were generated by the moderation team and alliances within the university. It would improve future reporting if it was possible to track the number of unique student posts. By July 2020 the top engagers in the group were student members. Overall engagement rates trended upwards during critical times in the university lifecycle, starting with a post-campaign launch engagement rate of 3\%, peaking in October at $41 \%$, then $32 \%$ in November and falling away again to $6.4 \%$ in December. 
Table 1.

Communications dashboard data monthly averages (May to December 2020)

\begin{tabular}{|l|l|}
\hline Engagement rate & $20 \%$ (cf. higher education industry average $0.2 \%)$ \\
\hline Number of posts & 68 \\
\hline Number of reactions & 1247 \\
\hline Number of comments & 281 \\
\hline
\end{tabular}

\section{Student participation}

The student voice was considered an integral part of the approach to engagement and creating a sustainable community. While most official RMIT student channels tend to 'broadcast' information about university services and events, RMIT Together is a group for students to connect and support each other. Even though moderating staff post into the group, the monthly data demonstrated that when students post content, engagement was higher. Moderators facilitated this in several ways and actively worked with student 'influencers' - e.g. by crafting posts to support R U OK? Day. Rather than staff posting events or opportunities for students, where possible they would ask for work areas to source a student willing to post about why they were planning to participate (see Table 2.). Lastly, when students posted in the group, moderators always responded with "likes" and words of encouragement, aiming to shape ongoing engagement.

Table 2.

Student voice: Comparing engagement with two posts promoting the same content.

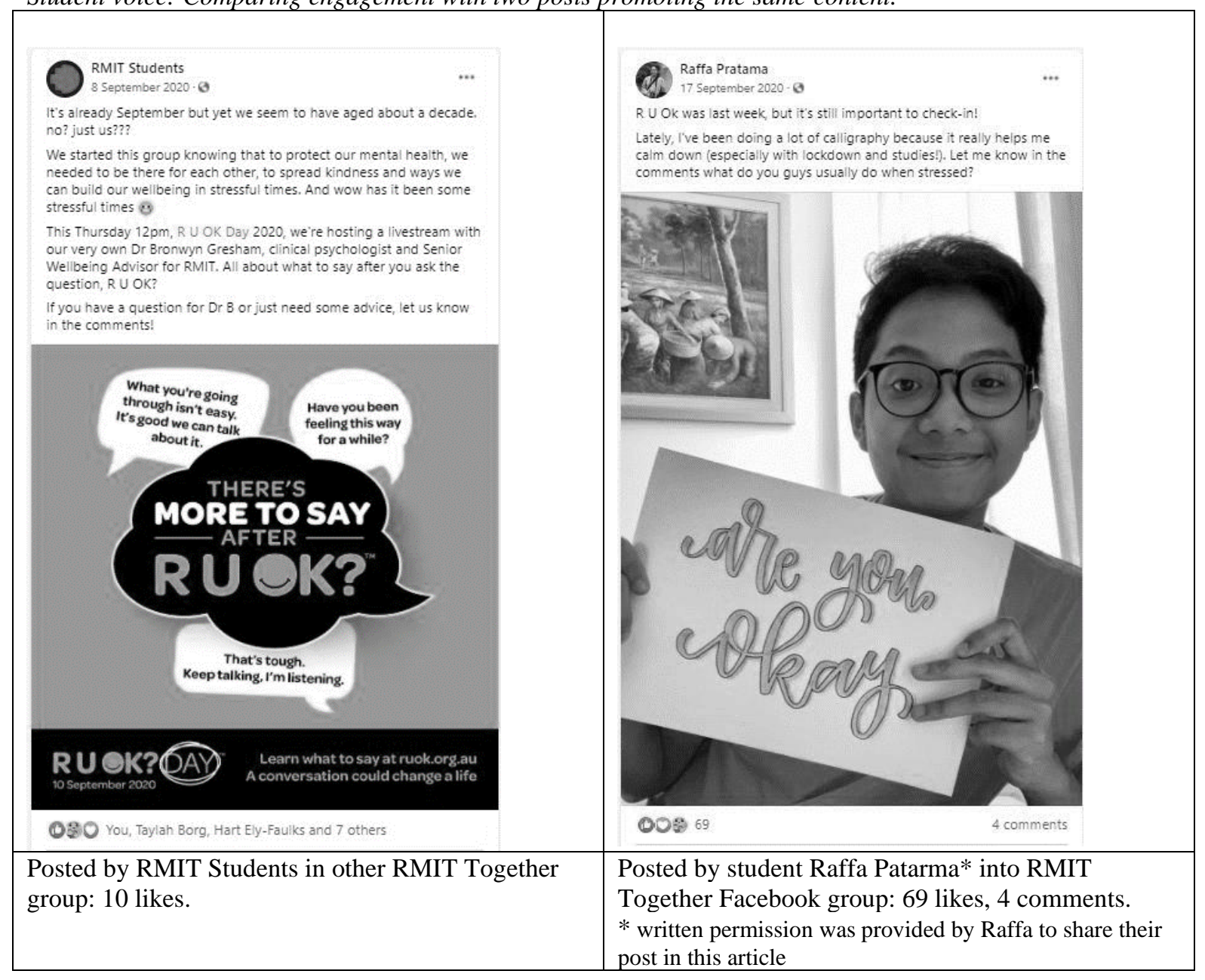




\section{Wellbeing Evaluation}

The wellbeing surveys conducted via the RMIT Together Facebook Group revealed information about the students in the group, wellbeing outcomes, and offered an opportunity for them to share feedback. Students were offered some incentive for completing the surveys, the chance to win a $\$ 50$ food voucher.

Nevertheless, response rates were low with $n=197$ semester 1 and $n=59$ semester 2 , so caution should be taken in interpreting the results too broadly.

Results across a selection of items from the two surveys were averaged demonstrating that:

- $69 \%$ of respondents were international students

- $60 \%$ reported studying at the postgraduate level,

- $28 \%$ were undergraduate students

- $7 \%$ were enrolled in vocational education

- $76 \%$ reported living in shared accommodation with family or friends

- $21 \%$ lived alone

Responses to the wellbeing questions suggest that students, or at least those who responded to the survey, were gaining benefit from engaging with the RMIT Together Facebook Group (Table 3).

Table 3.

RMIT Together Facebook group survey data

\begin{tabular}{|c|c|c|}
\hline & $\begin{array}{l}\text { Semester 1, } \\
2020 \\
\mathrm{~N}=197\end{array}$ & $\begin{array}{l}\text { Semester } 2 \\
2020 \\
\mathrm{~N}=59\end{array}$ \\
\hline Survey question & \multicolumn{2}{|c|}{$\%$ agree or strongly agree } \\
\hline This group has helped me understand more about my wellbeing & $77 \%$ & $88 \%$ \\
\hline $\begin{array}{l}\text { This group has encouraged me to take actions to care for myself } \\
\text { during my study. }\end{array}$ & $80 \%$ & $83 \%$ \\
\hline RMIT Together has helped me to feel connected during COVID-19. & $77 \%$ & $88 \%$ \\
\hline $\begin{array}{l}\text { I know where and how to seek support when concerned about } \\
\text { my/others wellbeing. }\end{array}$ & $84 \%$ & $99 \%$ \\
\hline $\begin{array}{l}\text { This group has supported me as I learn to adjust to the changes } \\
\text { COVID- } 19 \text { has made in my life. }\end{array}$ & $73 \%$ & $84 \%$ \\
\hline $\begin{array}{l}\text { I am using the Five Ways to Wellbeing model to support my study } \\
\text { life }\end{array}$ & NA & $70 \%$ \\
\hline
\end{tabular}

\section{Planning ahead}

While the global pandemic continues to impact students and their studies, RMIT Wellbeing will maintain the RMIT Together Facebook group (and accompanying web content) as a specialised mental health and wellbeing promotion hub.

For the Facebook group, 2021 brings a focus on streamlining content development, strengthening peer to peer engagement and student-led content generation. Ideas being explored include fostering RMIT Together student ambassadors who could take a leadership role in the group and offering work integrated learning placements.

The next phase will see the staff version of RMIT Together evaluated and reviewed. A year on from the beginning of the COVID-19 pandemic brings new challenges for both staff and students and the need for a refreshed focus. 


\section{Conclusion}

For staff and students, a preventative approach to mental health support has measurable impacts. By creating a clear strategy underpinned by evidence-based mental health models, shaping community participation and measuring impact, mental health promotion initiatives can have demonstrable benefits on student and staff mental wellbeing and may be particularly important for those at higher risk of mental ill health during drawn out stressful events like the COVID-19 pandemic. 


\section{References}

Aked, J., Marks, N., Cordon, C., Thompson, S., (2008). Five ways to wellbeing: A report presented to the Foresight Project on communicating the evidence base for improving people's wellbeing. Centre for wellbeing, The New Economics Foundation. United Kingdom Government, UK.

Aked, J., Thompson, S. (2011). Five ways to wellbeing: New applications, new ways of thinking. The New Economics Foundation. United Kingdom Government, UK.

Ebener, P. A., Hunter, S. B., Adams, R. M., Eisenman, D., Acosta, J. D., Chinman, M. (2017). Getting to Outcomes: guide for community emergency preparedness. RAND corporation, Santa Monica, Calif.

Mental Health Commission. (2020). National Mental Health and Wellbeing Pandemic Response Plan. Australian Government, ACT.

McGorry, P. Building the momentum and blueprint for reform in youth mental health. Lancet Psychiatry. 2019; 6(6): 459-61.

National Mental Health Commission (2020). We are \#inthistogether https://www.mentalhealthcommission.gov.au/news/2020/march/inthistogether-covid19.

Orygen. (2020). Australian University Mental Health Framework. Melbourne: Orygen 2020.

\section{The authors may be contacted via}

bronwyn.gresham@rmit.edu.au

\section{Please cite this paper as:}

Gresham, B., Garrick, V., Chee, L., Scott, C. (2021). RMIT together: An integrated mental health promotion campaign designed to build wellbeing and connection during the COVID-19 pandemic. Journal of the Australian and New Zealand Student Services Association, 29(1), 93-100. https://doi.org.10.30688/janzssa.2021.1.07 